\begin{tabular}{l|l} 
Investigaciones & $\begin{array}{l}\text { Revista Colombiana } \\
\text { de Educación, N. 62. } \\
\text { Primer semestre de 2012, } \\
\text { Bogotá, Colombia. }\end{array}$
\end{tabular}

\section{Rutas epistémicas y pedagógicas de la primera violencia en la enseñanza de las ciencias sociales: entre la memoria oficial y las otras memorias}

//Fpistemologv and pedagogy patterns

of the first violence by teaching Social

Science: Between official history and

other memoirs

//Rotas epistémicas e pedagógicas da primeira violência no ensino de ciências sociais: entre a memória oficial e as outras memórias

Jorge Enrique Aponte Otálvaro *

\begin{abstract}
Magister en Estudios Sociales de la Universidad Pedagógica Nacional y licenciado en Educación Básica con Énfasis en Ciencias Sociales de la Universidad Distrital Francisco José de Caldas. Docente-investigador de la Universidad Pedagógica Nacional, vinculado a los grupos de investigación Sujetos y nuevas narrativas en investigación y enseñanza de las ciencias sociales, en la línea Memorias, identidades y actores sociales de la Universidad Pedagógica Nacional y Cyberia de la Universidad Distrital Francisco José de Caldas. Correo electrónico: jeaponteo@gmail.com
\end{abstract}

\section{Resumen}

Este documento presenta el desarrollo y conclusiones de la investigación realizada por el grupo Cyberia en la línea investigativa de memoria y conflicto del Instituto para la Paz, la Pedagogía y el Conflicto de la Universidad Distrital (Ipazud), titulado "La primera violencia en la enseñanza de las ciencias sociales, entre la memoria oficial y otras memorias", Ilevado a cabo de noviembre de 2007 a marzo de 2009 en seis instituciones educativas de Bogotá. El propósito de la investigación fue establecer el abordaje epistémico y pedagógico de la primera violencia en la enseñanza de las ciencias sociales, así como evidenciar el lugar de esta área en el debate actual entre la memoria oficial y otras memorias. En el proyecto participaron cuatro instituciones oficiales de la ciudad ${ }^{1}$ y dos instituciones privadas, todas de características diferentes.

El itinerario metodológico de la investigación tomó como base las configuraciones trazadas por el enfoque genealógico-arqueológico, a partir del cual se diseñaron cuatro estrategias de recolección y análisis de la información: investigación documental, análisis de contenido, observación no participante y entrevistas semiestructuradas.

\section{Palabras Clave}

Memoria oficial, otras memorias, primera violencia, enseñanza de ciencias sociales, prácticas de enseñanza. 


\section{Abstract}

This paper offers a development and some conclusions by Cyberia, a research group of Ipazud, following a research line of memoir and conflict at Universidad Distrital, called 'The first violence by teaching Social Science: between official history and other memoirs', which took place from November 2007 to March 2009 in six Bogota schools. The research goal was to determine the epistemological and pedagogical approach of first violence by teaching Social Science as well as proving the position of this area in the current debate between official history and other memoirs.

The methodological research route based on standards given by a genealogicaland-archeological focus in order to implement four strategies of data collection and analysis -documentary investigation, contents analysis, non-participating observation, and semi-structured interviews.

\section{Keywords}

Official history, other memoirs, first violence, teaching Social Science, teaching practices.

\section{Resumo}

Este documento apresenta o desenvolvimento e as conclusões da investigação realizada pelo grupo Cyberia na linha investigativa de memória e conflito do Instituto para la Paz, la Pedagogía y el Conflicto da Universidad Distrital (Ipazud), intitulado "La primera violencia en la enseñanza de las ciencias sociales, entre la memoria oficial y otras memorias", realizado de novembro de 2007 a março de 2009 em seis instituições educativas de Bogotá. O propósito da investigação foi estabelecer a abordagem epistémica e pedagógica da primeira violência no ensino de ciências sociais, assim como evidenciar o lugar desta área no debate atual sobre a memória oficial e outras memórias. Participaram do projeto quatro instituições oficiais da cidade e duas instituições privadas, todas de características diferentes. O itinerário metodológico da investigação teve como base as configurações traçadas pelo enfoque genealógico-arqueológico, a partir do qual foram desenhadas quatro estratégias de coleta e análise da informação: investigação documental, análise de conteúdo, observação não participante e entrevistas semiestruturadas.

\section{Palavras chave}

Memória oficial, outras memórias, primeira violência, ensino de ciências sociais, práticas de ensino. 
Este artículo se inscribe en las discusiones sobre los estudios de la memoria y la historia social de currículo. De una parte, porque tiene la intención de presentar en el ámbito investigativo de la memoria social elementos analíticos que permitan comprender cómo, en escenarios de disputa, la memoria social es reglada y determinada en su producción discursiva. De otro lado, aborda el desarrollo de un área de conocimiento escolar que a la vez que incide en los procesos de producción de sentidos del pasado también representa los procesos de disputa por la conformación de la memoria social.

\section{Relaciones tensionales entre la memoria y la enseñanza de las ciencias sociales}

La memoria es una categoría emergente en las ciencias sociales del país. Su irrupción ha permeado diferentes escenarios investigativos y académicos ${ }^{1}$. Tal es el caso del ámbito

\footnotetext{
Los estudios sobre la memoria y las luchas reivindicativas asociadas a este ámbito, comienzan a ser objeto profuso de indagación y análisis en el contexto organizativo y académico colombiano. En el ámbito académico son notorios los esfuerzos en la creación de programas investigativos así como de líneas temáticas y de formación en programas universitarios. En este ámbito, valga señalar las producciones académicas de la línea de formación política y reconstrucción de la memoria social, adscrita al Departamento de Ciencias Sociales de la Universidad Pedagógica Nacional; los adelantos investigativos de la línea de investigación "Memoria y conflicto" del Instituto para la Pedagogía, la Paz y el conflicto Urbano IPAZUD; la creación en el año 2007 de la Red de estudios de la Memoria; la creación del grupo Memoria y palabra en la Universidad
}

escolar, en el cual la irrupción de los temas relacionados con la memoria del pasado reciente de la nación ha propiciado nuevos problemas y temáticas en el ámbito de la enseñanza, en especifico de las ciencias sociales escolares.

En este ámbito, la investigación adelantada por el grupo de investigación Cyberia estableció que la enseñanza de la Historia escolar, en lo que atañe a la primera violencia en Colombia ${ }^{2}$, se ha convertido en un dispositivo de reproducción de la memoria oficial sobre los acontecimientos que rodearon el desarrollo de la violencia política durante la primera mitad del siglo XX. De allí que se constituya en instrumento para la exclusión de otros relatos y memorias que emergen sobre el mismo fenómeno. Por tal motivo la investigación buscó develar un proceso de construcción y reconstrucción de la memoria colombiana sobre la primera Violencia, incluyendo las voces silenciadas y marginadas.

Nacional, la formación del centro de memoria paz y reconciliación adscrito a la administración distrital de Bogotá. Al respecto ver: Rodríguez Ávila, S. (2009). Jiménez y Guerra (Eds.) (2009). Rodríguez y Acosta (2008). Rodríguez y Sánchez (2009). Girón (2007), Aponte [Et al.] (2009), Silva [Et al.] (2010).

2 La categoría de primera Violencia emerge al interior de la historiografía sobre la violencia en Colombia como el periodo comprendido entre 1946 a 1948, marcados por el fin de la hegemonía liberal y el inicio de la hegemonía conservadora tras el triunfo de Mariano Ospina Pérez en las elecciones presidenciales; como tal el periodo se cierra con el asesinato de Jorge Eliecer Gaitán y se caracteriza por el recrudecimiento de las persecuciones a los miembros del Partido Liberal y el movimiento gaitanista. Ver: Guzmán, G., Fals, O., y Umaña, E. (2005), Green, J. (2003). Gaitán, G. (1985). Cárdenas M. (Ed.). 
En este proceso, se consolidó la discusión alrededor de las practicas de enseñanza de las ciencias sociales en la educación básica, en la medida en que se exploraron herramientas de renovación epistémicas, metodológicas y políticas para la enseñanza de la primera violencia en Colombia, examinando diversas fuentes de producción de discurso sobre la primera violencia y promoviendo su inclusión en la esfera escolar.

De igual forma, se tomó en cuenta el papel de la escuela como agente de construcción y producción de memoria colectiva desde una perspectiva crítica, que busca la inclusión de múltiples memorias a la hora de conformar las representaciones del pasado como una de las funciones centrales de la enseñanza de la historia y de las ciencias sociales.

El objetivo central de la investigación fue determinar cómo se aborda, epistémica y pedagógicamente, la primera violencia en la enseñanza de las ciencias sociales, en la educación básica en Bogotá. Ello a través de evidenciar la posición de la enseñanza de las ciencias sociales en el marco del debate actual entre la memoria oficial y otras memorias en relación a la problemática de la primera violencia. Así mismo, se dio cuenta del tratamiento actual de la primera violencia en la enseñanza de las ciencias sociales, analizando la normatividad educativa y libros de texto del área, de la mano del análisis de la existencia o no de otras memorias y la forma como se aborda actualmente la primera violencia en las prácticas de enseñanza de las ciencias sociales en seis colegios de educación básica de Bogotá.

\section{Proceso metodológico: descripción enunciativa del discurso}

El recorrido metodológico tuvo como soporte los referentes teóricos del enfoque genealógico-arqueológico, desde el cual se ajustaron cuatro estrategias investigativas: investigación documental, análisis de contenido, observación no participante y entrevistas semiestructuradas.

Metodológicamente, se hizo el análisis a partir del enfoque genealógico-arqueológico, en tanto que se partía por comprender a las ciencias sociales escolares como un discurso. En dicho enfoque, el discurso es comprendido más allá de un conjunto de actuaciones verbales o signos, ya sea de forma escrita u oral, ampliando su visión a un conjunto de acontecimientos discursivos que responden a un 
sistema de formación determinada. Al respecto Foucault afirma: "El discurso está constituido por un conjunto de secuencias de signos, en tanto que esas son enunciados, es decir en tanto que se les puede asignar modalidades particulares de existencia (Foucault, 2005, p. 181).

Modalidades de existencia que hacen referencia a las formas, los tipos y las posibilidades de relación que pueden establecer los discursos y a su presencia material, es decir a las prácticas sociales que pueden llegar a constituir en la sociedad.

El análisis genealógico-arqueológico propende por "la descripción pura de los acontecimientos discursivos como horizonte para la búsqueda de unidades que en ellos se forman" (Foucault, 2005, p. 43). Dichas unidades se ciernen sobre el enunciado, el cual, dentro del enfoque, se presenta como el insumo esencial de los discursos y el cual se diferencia del enunciado en sentido gramatical o a su utilización por el análisis del discurso. El trabajo investigativo que se realiza desde un enfoque genealógico-arqueológico:

[...] trata de captar el enunciado en la estrechez y la singularidad de su acontecer; de determinar las condiciones de su existencia, de fijar sus límites de la manera más exacta, de establecer sus correlaciones con los otros enunciados que pueden tener vínculos con él, demostrar qué otras formas de enunciación excluye... se debe mostrar por qué no podía ser otro de lo que era, en qué excluye a cualquier otro, cómo ocupa en medio de los demás y en relación con ellos un lugar que ningún otro podría ocupar. La pregunta adecuada a tal análisis se podría formular así: ¿Cuál es, pues, esa singular existencia que sale a la luz en lo que se dice y en ninguna otra parte? (Foucault, 2005, p. 26).

El grupo de investigación Cyberia denomina este tipo de investigación como descripción enunciativa del discurso, proceso en el cual se establecieron tejidos relacionales a partir de los documentos y la información acopiada, los cuales permitieron establecer las condiciones particulares de los enunciados presentes en las prácticas de enseñanza de las ciencias sociales en relación con el fenómeno estudiado.

Para el caso de la investigación adelantada, se estableció que el archivo fueran documentos tales como diarios de campo de clases de ciencias sociales, entrevistas a estudiantes y profesores y programas de estudio, libros de texto y material didáctico de ciencias sociales que circulara en los colegios. En el análisis de este archivo se procedió a registrar grupos de enunciados sobre 
la primera violencia, la memoria oficial y la enseñanza de las ciencias sociales.

El examen documental del archivo mencionado se adelantó por medio de la implementación de varias matrices temáticas de análisis, a través de la cuales se organizó y sistematizó la información recabada en los documentos. Como resultado de este análisis se identificaron cuatro dominios discursivos, los cuales dan cuenta de la relación entre la memoria y la enseñanza de la primera violencia en las prácticas de enseñanza de las ciencias sociales.

\section{Resultados: campos tensionales en la relación memoria y enseñanza}

A partir del análisis del archivo recabado sobre la memoria de la primera violencia en instituciones escolares, se determinaron cuatro dominios discursivos (epistémico, pedagógico-evaluativo, formativo axiológico y político) al interior del campo de saber de la enseñanza de las ciencias sociales. Dominios que no son homogéneos y que manifiestan en su interior tensiones.

El primer dominio identificado, denominado epistémico, hace referencia a las concepciones que alimentan las representaciones del pasado sobre la primera Violencia. En este contexto se manifiesta una creciente tensión entre las posturas que constituyen el código disciplinar ${ }^{3}$, en las cuales la primera Violencia es reducida a un enfrentamiento bipartidista estableciendo su inicio en un punto fijo en el tiempo, preso de una figura (Jorge Eliecer Gaitán) y un acontecimiento (su asesinato el 9 de abril de 1948), y de igual forma su supuesta conclusión a través del establecimiento del Frente Nacional. Ante esta postura, se presentan memorias que representan el pasado de forma alternativa estableciendo lecturas de carácter estructural para el inicio de la Violencia, las figuraciones del pasado hechas desde la literatura, el cine y la música, así como la de los actores involucrados en conflictos, como el movimiento gaitanista.

3 La categoría Código Disciplinar fue propuesta por el profesor e investigador Raimundo Cuesta y la define como "[...] el conjunto de ideas, valores, suposiciones, reglamentaciones y rutinas prácticas (de carácter expreso o tácito). En suma, el elenco de ideas, discursos y prácticas dominantes en la enseñanza de las asignaturas dentro del marco escolar. El código disciplinar alberga, pues, las especulaciones y retóricas discursivas sobre su valor educativo, los contenidos de enseñanza y los arquetipos de práctica docente, que se suceden en el tiempo y que se consideran, dentro de la cultura, valiosos y legítimos" En: Cuesta, R. (1997, p. 33) 
En el dominio pedagógico, se analizó la manera como se abordan las conceptualizaciones de las ciencias sociales y el manejo de este saber en las prácticas de enseñanza, identificándose tres tensiones: la primera, entre la enseñanza tradicional y las prácticas innovadoras, que se limita a la implementación de herramientas didácticas, más no a un trabajo epistémico y político. La segunda tensión se localizó entre dos posturas: la memoria y la memorización. La memorización se caracteriza por el deseo de interiorizar, a través de procesos mnemotécnicos, una mirada apologética de la historia, resaltando próceres, fechas y acontecimientos específicos, manteniendo o reproduciendo la memoria oficial. Postura que se confronta con la elaboración de una memoria sobre hechos del pasado, narrativa que busca, por medio de la pedagogía, darle sentido a un pasado con referencia al presente. Así lo afirma una de las docentes entrevistadas a propósito de su práctica escolar:

Ellos [los estudiantes] todavía tienen problemas para entender cuando la historia no se les da lineal, entonces cuando hacemos las relaciones de contexto, les cuesta trabajo. Cuando uno se va a otro país o cuando, por ejemplo, veíamos algo que les costaba trabajo como revolución neolítica, la revolución agrícola, la revolución industrial y la colonización antioqueña, para que ellos entiendan las condiciones que generan un cambio [...] cuesta trabajo, porque si no se aprenden todo de memoria, ahí sí de memoria, y lo importante es que ellos puedan relacionar los elementos comunes, así sean épocas muy diversas (entrevista docente, 17 de octubre de 2008).

La tercera tensión se presenta entre los enfoques pedagógicos y las prácticas del aula de clase o también denominadas prácticas de enseñanza. En esta tensión se logró establecer cómo las prácticas al interior del aula siguen estando sujetas a un proceso tradicional de evaluación como el examen y el taIler, convirtiendo aprendizajes, de una u otra forma enriquecedores, constructivos y bien estructurados, en aprendizajes lineales y con un origen único e histórico.

En el dominio discursivo de lo pedagógico-evaluativo se estableció que existe un énfasis formativo que recae en la enseñanza de las ciencias sociales, en un enfoque que orienta el horizonte epistémico hacia una labor que compromete el campo axiológico, el cual se establece como dominio discursivo. Desde esta perspectiva, la enseñanza de la primera violencia se centra en el establecimiento de dinámicas 
de exposición de eventos ejemplarizantes que sirvan de modelo para orientar la acción de los sujetos en la sociedad, desde una perspectiva de convivencia pacífica.

[...] cuando yo abordo el tema, la primera pregunta de los niños es "profesora ¿será que vuelve a suceder una guerra de esas ahora que nosotros estamos vivos?", eso me preguntaron, y les dije que en este momento el ser humano es responsable de que eso no vuelva a suceder, porque si nosotros tenemos la voluntad y la disposición de dialogar y de concertar, eso nunca va a volver a suceder, nosotros siempre tenemos que buscar impulsar este tipo de valores para que los niños siempre los estén practicando (Entrevista docente, 11 de septiembre de 2008).

En tal sentido, se pretende que las representaciones del pasado, lejos de constituirse en herramientas para el análisis estructural de la realidad circundante de manera crítica, se conviertan en elementos que normalizan a los sujetos, haciendo ver el conflicto como algo indeseable. La tensión se afinca entre la formación de "ciudadanos" acríticos pacíficos y la posibilidad de constituir sujetos críticos activos, entre la memoria como fuente ejemplarizante o herramienta para la reflexión crítica. Posturas que se muestran en afirmaciones como esta:

Para ciencias sociales se está trabajando este año fuertemente en el desarrollo de los derechos humanos, también en el desarrollo de la tolerancia, de la diversidad, de los valores en cada una de las clases. Independientemente si es ética, religión, sociales o economía, se les está formando de manera integral en eso (Entrevista docente, 29 de septiembre de 2008).

[...] lograr que los chicos se acerquen a una realidad, específicamente ahora a unas realidades de su entorno como país, como nación y Estado, y de cómo ellos contribuyen como ciudadanos. Si uno tiene como fin eso, pues toda su labor como docente se va a centralizar y orientar hacia qué hace uno como maestro para llevar a mostrarle a los chicos esos mundos, qué hace uno para fortalecer ciertas actitudes y ciertos valores ciudadanos, valores como la participación, la reflexión y la conciencia ciudadana (Entrevista docente, 10 de septiembre de 2008). 
El último de los dominios discursivos explorados hace frente al uso de la memoria de la primera Violencia como instrumento para la orientación de la acción política de los sujetos, la cual se desarrolla en dos niveles entre los cuales se manifiesta tensión. El nivel micro hace referencia a la orientación de la acción con arreglo a criterios de comportamiento que eviten la resolución de conflictos de manera violenta, acudiendo a los marcos formales de la democracia. Orientación en la cual la primera Violencia es tratada a través de cada uno de sus episodios ejemplarizantes, careciendo de una lectura de contexto de los mismos.

[...] la actual [violencia] arranca desde la muerte de Gaitán, gracias a que los sectores hegemónicos en la estructura económica y política sabían que Gaitán era un problema para sus intereses, por eso lo mataron. De allí arrancan a matar a los campesinos para apoderarse de sus tierras, por eso se forman las guerrillas y no simplemente porque sí como lo muestran los medios de comunicación, eso es lo que deben entender los estudiantes, para pensar bien a la hora de votar (Entrevista docente, 11 de septiembre de 2008).
Por otra parte, el nivel macro hace referencia a la orientación de la acción de los sujetos a la hora de participar en la determinación de los destinos de los colectivos a los cuales pertenecen.
Las nuevas generaciones tienen que ir aprendien- do todos estos hechos que han venido pasando a lo largo de la historia de este país para poder, más adelante, participar de la vida política basándose en estos hechos, enten- der cómo estos se refle- jan hoy en día (entrevista docente, 15 de octubre de 2008).

Esta orientación busca que los estudiantes, a la hora de ejercer su derecho a la elección de gobernantes, se inclinen por aquellos proyectos que brinden alternativas al conflicto armado, en contra de sectores políticos que pretenden terminar con el conflicto por medio del recrudecimiento de la guerra. Así lo evidencia el uso de la violencia como eje de análisis y de lecturas criticas frente a gobiernos de la época.

$$
\begin{aligned}
& \text { [...] yo había pensado } \\
& \text { partir de lo que eran los } \\
& \text { conflictos de los partidos } \\
& \text { políticos, a raíz de algo } \\
& \text { que estaba sucediendo a } \\
& \text { principios de año y que } \\
& \text { hoy tiene que ver con } \\
& \text { el uribismo, pero nun- } \\
& \text { ca tuvo mucha acogida, }
\end{aligned}
$$


entonces empezamos desde el desplazamiento, con el otro nivel el año antepasado, con toda la situación de la reelección de Uribe y las elecciones, entonces empezamos a abordar desde los partidos políticos, lo cual nos llevó a observar el conflicto colombiano más desde esos planteamientos ideológicos y de esos conflictos oligarcas (Entrevista docente, 10 septiembre de 2008).

\section{Conclusiones: la institución escolar como escenario de luchas por el pasado}

Al interior del campo de saber de la enseñanza de las ciencias sociales y de los cuatro dominios discursivos identificados, así como de sus tensiones, se puede evidenciar la preeminencia del componente evaluativo y axiológico en detrimento de la ampliación de las fuentes que alimentan el campo epistémico y político.

El uso político de la memoria en la escuela se desenvuelve en dos escenarios: por una parte, el uso de datos ejemplarizantes para la adaptación de los sujetos al sistema institucional y por otra, la resistencia de algunos maestros, los cuales a través de su práctica pedagógica permiten la emergencia de distintas representaciones del pasado en la tarea de formar sujetos críticos que orienten su acción política en el nivel macro, para diseñar y elegir proyectos colectivos que se ocupen de afrontar las causa estructurales de la violencia.

Sin embargo, la investigación evidenció la emergencia de otras narrativas que agencian memoria sobre la primera Violencia en Colombia. Tal es el caso del uso de algunos maestros de fuentes como el cine, la literatura, la música, la historia oral, la pintura y la fotografía como herramientas para la representación del pasado, aunque siguen sometidas, a la comprensión tradicional del asesinato de Jorge Eliécer Gaitán como principal causa de la Violencia.

La institución escolar es un campo de lucha en el que la práctica de enseñanza de las ciencias sociales se ejerce en medio de distintos campos de fuerza, saber y poder, donde se disputa el dominio estratégico de instituciones, cuerpos y conciencias. 


\section{Referencias}

Aponte, J., Silva, O., Cano, P., Díaz, D., Martínez, N., Pinto, J. y Pineda, A. (2009). Memoria oficial y otras memorias: la disputa por los sentidos del pasado. Ciudad Paz-ando, 3, 203-218.

Gaitán, G. (1985). Los orígenes de la violencia de los años 40. En: Cárdenas M. (Ed.), Once ensayos sobre la Violencia. Bogotá: Centro Gaitán; Fondo Editorial CEREC.

Green, J. (2003). Gaitanismo, Left Liberalism and Social Mobilization in Colombia. [Gaitanismo, izquierda liberal y movilización social en Colombia]. GainesviIle: University Press of Florida.

Guzmán, G., Fals, O., y Umaña, E. (2005). La Violencia en Colombia. Tomo I. Bogota: Taurus.

Cuesta, R. (1997). Sociogénesis de una disciplina escolar: la Historia. Barcelona: Ediciones Pomares-Corredor, S.A.

Dema, P. (2008). El relato literario y la memoria colectiva [Versión electrónica]. Revista Borradores, 8 y 9, Extraído el 27 de mayo de 2008 de http://www. unrc.edu.ar/publicar/borradores/Vol89/pdf/Elrelatoliterarioylamemoriacolectiva.pdf

Finocchio, S. (1993). Enseñar ciencias sociales. Buenos Aires: Editorial Troquel.

Foucault, M. (1980). La verdad y las formas jurídicas. Barcelona: Gedisa. [Versión electrónica] Ex- traído el 15 de mayo de 2008 de http://www.pensamientopenal. com.ar/04042008/filosofia02. pdf

Foucault, M. (1983) El sujeto y el poder [Versión electrónica]. Extraído el 18 de febrero de 2008 de http://www.philosophia.cl/ biblioteca/Foucault/El\%20sujeto $\% 20 y \% 20$ el\%20poder.pdf

Foucault, M. (1993). Las palabras y las cosas. Una arqueología de las ciencias humanas. Argentina: Siglo XXI Editores.

Foucault, M. (2005). La arqueología del saber. México: Siglo XXI Editores.

Foucault, M. (2007). Microfísica del poder [Versión electrónica] Extraído el 18 de marzo de 2008 de http://caosmosis.acracia.net/ wp-content/uploads/2007/11/ foucault-microfisica-del-poder. pdf

Girón O, C. (2007). Colombia: la pedagogía social de la memoria histórica. En: Revista Puentes. Año 7 No 22, pp. 46-50.

Jelin, E. y Lorenz, F. (2004). Educación y memoria. La escuela elabora el pasado. Madrid: Siglo Veintiuno de España editores.

Ricoeur, P. (1998). La lectura del tiempo pasado: memoria y olvido. España: Arrecife.

Ricoeur, P. (2004). La memoria, la historia, el olvido. México: Fondo Cultura Económica.

Rodríguez, S. (2009). El 9 de abril en 
las políticas de la memoria oficial: el texto escolar como dispositivo de olvido. En: Ayala Diago, C., Casallas Osorio, O., y Cruz Villalobos, H. (Eds.). Mataron a Gaitán 60 años. Pp. 135-154. Bogotá: Universidad Nacional de Colombia.

Rodríguez, S. (2009). Producción, difusión y consolidación de la memoria oficial en Colombia: 1930-1950'. En: Jiménez Becerra, A., y Guerra García, F. (Eds.). Las luchas por la memoria. pp. 95-125. Bogotá: Instituto para la pedagogía, la paz y el conflicto urbano (IPAZUD) y Universidad Distrital Francisco José de Caldas.

Rodríguez, S. y Acosta, W. (2008). Autores, problemas y debates en la investigación sobre la enseñanza de la historia (19902006). En: Memorias X jornadas nacionales y I internacional de Enseñanza de la Historia. Río Cuarto (Argentina): Asociación de Profesores de Enseñanza de la Historia de Universidades Nacionales (APEHUN).

Rodríguez, S. y Sánchez, O. (2009). Problemáticas de la enseñanza de la historia reciente en Colombia: trabajar con la memoria en un país en guerra. En: Revista Reseñas. № 7. Argentina: APEHUN.

Sánchez, G.( 2006). Guerras, memoria e historia. Bogotá: La Carreta Editores.

Serna, A. (2007). Sobre la memoria y el conflicto. En: Serna, A., Gómez, D. \& Guerra, F. Pedagogía, paz y conflicto: orientaciones para la docencia, la extensión y la investigación. (pp.75-168). Bogotá: Universidad Distrital Francisco José de Caldas.

Silva, O. [Et al.] (2010). Entre la memoria oficial y otras memorias: disputas de saber poder en la enseñanza de las ciencias sociales. La primera violencia en Colombia. Bogotá: Instituto para la pedagogía, la paz y el conflicto urbano (IPAZUD) y Universidad Distrital Francisco José de Caldas.

Traverso, E. (2007). El pasado: instrucciones de uso. Historia, memoria, política. Barcelona: Marcial Pons. 